\title{
1 A comprehensive co-expression network analysis in Vibrio cholerae
}

2 Cory D. DuPaia, Claus O. Wilke ${ }^{\mathrm{a}, \mathrm{b}}$, and Bryan W. Daviesa,

4 a Institute for Cellular and Molecular Biology, The University of Texas at Austin, Austin, TX,

5 USA

6 b Department of Integrative Biology, The University of Texas at Austin, Austin, TX, USA

$7 \quad$ c Department of Molecular Biosciences, The University of Texas at Austin, Austin, TX, USA

9 Abstract

10 Research into the evolution and pathogenesis of Vibrio cholerae has benefited greatly from

11 the generation of high throughput sequencing data to drive molecular analyses. The steady

12 accumulation of these datasets now provides a unique opportunity for in silico hypothesis

13 generation via co-expression analysis. Here we leverage all published V. cholerae RNA-

14 sequencing data, in combination with select data from other platforms, to generate a gene

15 co-expression network that validates known gene interactions and identifies novel genetic

16 partners across the entire $V$. cholerae genome. This network provides direct insights into

17 genes influencing pathogenicity, metabolism, and transcriptional regulation, further

18 clarifies results from previous sequencing experiments in V. cholerae (e.g. Tn-seq and ChIP-

19 seq), and expands upon micro-array based findings in related gram-negative bacteria.

\section{Importance}

22 Cholera is a devastating illness that kills tens of thousands of people annually. Vibrio

23 cholerae, the causative agent of cholera, is an important model organism to investigate both 
24 bacterial pathogenesis and the impact of horizontal gene transfer on the emergence and

25 dissemination of new virulent strains. Despite this importance, roughly one third of $V$.

26 cholerae genes are functionally un-annotated, leaving large gaps in our understanding of

27 this microbe. Through co-expression network analysis of existing RNA-sequencing data,

28 this work develops an approach to uncover novel gene-gene relationships and

29 contextualize genes with no known function, which will advance our understanding of $V$.

30 cholerae virulence and evolution.

\section{Introduction}

33 Since the completion of the first Vibrio cholerae genome sequence in 2000, over a

34 thousand $V$. cholerae isolates have been sequenced $(1,2)$. These sequences has allowed for

35 the development of sophisticated phylogeographic models, which emphasize the

36 importance of controlling the spread of virulent and antibiotic resistant $V$. cholerae strains

37 to lower disease burden, in addition to fighting endemic local strains (2-6). The integration

38 of hundreds of genomes paired with temporal and geographic information into ever

39 growing phylogenies enables analyses using selection models to predict future population

40 trends and derive biologically meaningful insights into V. cholerae evolution $(7,8)$. By

41 developing treatment and vaccination strategies based on phylogenic models (9),

42 organizations and governments can more efficiently leverage limited resources and more

43 effectively prevent disease spread in line with the World Health Organization's goal of

44 eradicating cholera by 2030 (10).

45 Alongside advances in genomics research, the $V$. cholerae and broader bacterial biology

46 communities have benefited greatly from other next generation sequencing (NGS) 
47 technologies. Targeted sequencing experiments have been essential in mapping complex

48 virulence pathways, illuminating a novel interbacterial defense system, and expanding our

49 knowledge of the role of non-coding RNA (ncRNA) in the vibrio life cycle (11-17). Further

50 discoveries such as transcription factor mediated transposon insertion bias (18) and the

51 role of cAMP receptor protein in host colonization (19) have benefited from composite

52 research strategies utilizing multiple technologies. Similarly, meta-analyses utilizing pooled

53 data from multiple experiments are empowered by the increasing availability of high

54 quality bacterial NGS datasets. Expression data is particularly amenable to such pooling

55 and can be used to accurately group genes into functional modules based on their co-

56 expression (20). In bacteria, weighted gene co-expression network analysis (WGCNA) (21)

57 has been successfully used to underscore biologically important genes and gene-gene

58 relationships via "guilt-by-association" approaches $(22,23)$. These studies have taken

59 advantage of larger and larger heterogeneous microarray datasets to provide novel

60 biological insights via existing data.

61 Despite major advances in sequencing technologies and research strategies, most of the

62 over two dozen existing RNA-seq experiments in $V$. cholerae have been limited to targeted

63 approaches that involve quantifying the differential abundance of genetic material across a

64 handful of conditions. Via these approaches, any change in expression observed in one

65 experiment is nearly impossible to generalize to other treatment conditions and analyses

66 are limited to a few pathways or genes of interest. In contrast, meta-analyses such as

67 WGCNA can uncover much broader relationships throughout the entire genome by

68 combining information from multiple datasets. As there is no existing co-expression

69 analysis in $V$. cholerae to date, the accumulation of over 300 publicly available RNA-seq 
70 samples from targeted RNA-seq experiments represents a heretofore untapped resource

71 for the cholera community.

$72 \quad$ Motivated by the success of pooled genetic sequencing analyses, our current work

73 utilizes all publicly available $V$. cholerae RNA-seq based expression-level data to generate a

74 co-expression network. We expand upon existing bacterial WGCNA approaches by

75 integrating broader sequencing data (including ChIP-seq and Tn-seq) and multiple

76 annotation platforms into our analysis. Our network ultimately contributes information on

77 connections across all $V$. cholerae genes, including the roughly 1500 predicted but

78 functionally un-annotated genetic elements that account for some $37 \%$ of the genome.

79 More specifically, we implicate new loci in virulence regulation and clearly demonstrate a

80 powerful and accurate approach to hypothesis generation via our described network.

82 Results

\section{Gene network generation}

84 To generate our co-expression analysis in $V$. cholerae, we applied our WGCNA pipeline to

85 analyze twenty-seven $V$. cholerae RNA sequencing experiments deposited in NCBI's

86 Sequence Read Archive (SRA) in addition to two novel experiments. The RNA sequencing

87 samples are derived from experiments exploring a range of important $V$. cholerae processes

88 including intestinal colonization, quorum sensing, and stress response. In total, our

89 network includes 300 individual RNA-seq samples (supplementary table S1). All samples

90 were mapped to a recently inferred $V$. cholerae transcriptome derived from the N16961

91 reference genome $(1,13)$. This reference was chosen because the majority (293) of

92 samples were collected from strains N16961 or the closely related C6706 and A1552. 
93 Figure 1 outlines the process used to generate our co-expression network with a small

94 subset of genes. Loci VC0384-VC0386 are known to be involved in cysteine metabolism

95 while the two genomically adjacent loci VC0383 and VC0388 do not share this function.

96 Following normalization of mapped transcripts (Fig. 1A), a weighted gene co-expression

97 network analysis was performed using WGCNA (21). First, a Pearson correlation matrix is

98 calculated for expression levels of all genes (Fig. 1B). This correlation matrix clearly

99 captures strong relationships between co-expressing genes such as VC0384-VC0386 but

100 can produce background noise from un-related gene pairs. We limit this noise by

101 calculating a topological overlap matrix (TOM) (24) that weights pairwise co-expression

102 data based on each gene's interactions with all other genes (Fig. 1C). In this way, the

103 relationships between genes that fall within the same subnetwork, i.e. VC0384-86, are

104 favored while the signal from unrelated genes, i.e. VC0383 and VC0388, is abated. This

105 TOM, after filtering for normalized values greater than 0.1, is used to construct an accurate

106 co-expression network that captures biologically meaningful relationships (Fig. 1D).

107 In addition to co-expression data, our network and analyses incorporate information

108 from multiple other sources. Our network includes predicted pathway annotations and

109 gene functional knowledge from the NCBI Biosystems database as well as the DAVID,

110 Panther, and KEGG databases (25-28). Additionally, importance labels are applied to genes

111 with no known function which have been implicated as playing a role in intestinal

112 colonization or in vitro growth via Tn-seq based essentiality experiments $(14,29)$.

113 Information from ChIP-seq binding assays and microarray results were incorporated in

114 downstream analyses to substantiate network derived relationships. By combining all of

115 these data sources we were able to develop and analyze an informative network of co- 
116 expressing genes that provides both qualitative and quantitative information about

117 relationships between transcripts across forty-nine gene-clusters covering the entire $V$.

118 cholerae genome (Supp. Data S1-2).

120 Genes in known pathways cluster together and contextualize genes of unknown

\section{1 function}

122 As proof of the accuracy of our approach, we have highlighted four clusters that

123 recapitulate known interactions between transcripts involved in specific pathways or

124 cellular processes (Fig. 2). The correct grouping of transcripts encoding products such as

125 ribosomal proteins, amino acid synthesis proteins, and tRNA transcripts that have largely

126 known functions and are involved in well-studied, highly conserved cellular processes

127 provides a positive control for the validity of our network clusters (Fig. 2A-C). Likewise,

128 the clustering of genes known to be involved in more specialized processes such as biofilm

129 formation (Fig. 2D) further underscores the success of our approach.

130 The subnetworks mentioned above also support the utility of our analysis in powering

131 guilt-by-association based inference of gene function (30). Because each of these gene

132 clusters contain co-expressing genes that are involved in the same biological process, it can

133 be assumed that unannotated genes in the same cluster are likely involved in the same

134 process. Such links, while not definitive on their own, can be used with other data to hint at

135 gene functions. For example, genes with known function in Fig. 2D are primarily involved

136 in biofilm formation $(31,32)$. This clustering of biofilm genes suggests that the few genes

137 with no known function in this subnetwork may be involved in the same process. Two of

138 these unannotated transcripts, VC1937 and VC2388, are, per GO cellular component 
139 location labels, "integral membrane components." Further, the VC2388 locus is directly

140 upstream of a Vcr084, a short RNA involved in quorum sensing which is essential for

141 biofilm formation (33). Taken together, this evidence suggests that VC1937 and VC2388

142 may play a role in some of the complex membrane restructuring necessary for biofilm

143 formation. In facilitating such guilt-by-association approaches to novel hypothesis

144 generation, our co-expression network serves as a highly efficient substitute for more

145 traditional screening assays.

\section{A virulence subnetwork suggests novel gene functions}

148 While the biofilm associated subnetwork presents a relatively simple example of the

149 functional insights our co-expression data can yield, the virulence-related subnetwork (Fig.

$1503 \mathrm{~A}$ ) represents a more complex case in which genes of known function provide clues to the

151 role of unannotated genes. The majority of transcripts in this module originate from within

152 the virulence-related ToxR regulon that consists principally of genes on the $V$. cholerae

153 pathogenicity island 1 (VC0809-VC0848) and cholera toxin sub-units A and B (ctxAB,

154 VC1456 and VC1457) (34). Other genes in this subnetwork, such as vpsJ, VC1806, VC1810,

155 and chitinase, are predominately localized to virulence islands and other areas of the

156 genome under tight control of the known virulence regulators ToxR, ToxT, or H-NS as

157 determined via ChIP and/or RNA-seq (35-37). The clustering of such genes with well-

158 characterized interactions into a cohesive subnetwork is further validation of our ability to

159 generate accurate co-expression maps of related genes. The association of uncharacterized

160 genes in these clusters suggests they may also play a role in $V$. cholerae virulence and

161 generates hypotheses about the function of unknown genes within this module. 
162 Many of the important transcripts with unknown function are expected to co-express

163 with known virulence genes because they fall within vibrio pathogenicity island (VPI)-1

164 (VC0810, VC0821-VC0823, VC0842) or VPI-2 (VC1806, VC1810), or are proximal to other

165 virulence genes (VC1945) $(38,39)$. However, our analysis also identified genes such as

166 VCA0094-VCA0096 which are on a completely different chromosome than the rest of the

167 subnetwork and do not neighbor any known virulence elements.

168 A major benefit of our approach is that we incorporate additional regulatory data such

169 as ChIP and Tn-seq into our co-expression analysis, allowing us to verify the association

170 between VCA0094-VCA0096 and virulence pathways using existing experimental data. Tn-

171 seq analysis has previously identified VCA0094 and VCA0095 as essential for infection of a

172 rabbit intestine (14), suggesting that these loci play a role in virulence. Because transcripts

173 for these genes co-express with genes regulated by ToxT, ToxR, and H-NS, we also probed

174 existing ChIPseq binding datasets $(12,19,35)$ to see if any of these well-studied

175 transcription factors bind near the VCA0094-96 loci. While ToxT binding was not observed

176 near this site (data not shown), our analysis identified significant peaks in the promoter

177 region of VCA0094 for both ToxR and H-NS as calculated via re-analysis of existing binding

178 data from (35). Both peaks showed a large and significant increase in binding affinity $\left(\log _{2}\right.$

179 fold change in average occupancy) when compared against input controls (Fig. 3B). H-NS

180 showed a clear binding peak in the region of the VCA0094 promoter that extended in a

181 diffuse manner to the VCA0095 TSS while ToxR binding covered a similar region but was

182 more diffuse throughout (data not shown). Collectively these results indicate virulence

183 related functions for the products of the VCA0094-VCA0096 transcripts. Although the

184 exact mechanistic role of these genes remains elusive, we have nevertheless demonstrated 
185 the ability of our pipeline to generate meaningful hypotheses by incorporating existing data

186 from a multitude of sources.

\section{Co-expression data provides an accurate in silico complement to RNA-seq}

189 In addition to the guilt-by-association inference described above, co-expression analysis

190 can provide a partial substitute or complement to RNA-seq experiments. Novel, meaningful

191 genetic relationships can be found in a co-expression network by focusing on the

192 transcripts that are co-regulated with a gene of interest.

193 We can apply a network-based approach in lieu of new RNA-seq based experiments to

194 identify genes which co-express with $r p o S$ (VC0534) and are similarly involved in bacterial

195 stress response. As our network utilizes only RNA-seq based transcriptomics studies and

196 none of these studies involves direct manipulation of $r p o S$, we can compare existing

197 microarray data involving an $\operatorname{rpoS}$ (VC0534) deletion mutant (40) to determine how

198 accurate our approach is. When applying an absolute co-expression cutoff of $0.1,273$ genes

199 are identified as having a relationship with $r p o S$ expression in both our network analysis

200 and the rpoS mutant microarray data (Fig. 4A). This represents nearly two-thirds of genes

201 identified as differentially expressed in the original microarray study. While our network

202 links far more genes with rpoS than the microarray approach, this is in line with recent

203 RNA-seq based work that found that $23 \%$ of the E. coli genome is regulated by RpoS (41).

204 Additionally, all of the flagella and chemotaxis related proteins highlighted as particularly

205 informative in the original study are identified by our analysis (Fig. 4B) and relevant values

206 (i.e. network co-expression and microarray-derived log fold change in expression) for the

207273 shared transcripts have a Spearman correlation of -0.516 . This accuracy is achieved 
208 without any direct genetic manipulation of the rpoS locus in the RNA-seq datasets used to

209 generate our co-expression network and serves as a testament to the potential utility and

210 versatility of our approach.

211 Our approach to isolating genetic interactions also has advantages over

212 transcriptomics-focused sequencing. As seen in Fig. 4A, our network-based analysis

213 identifies far more genes associated with rpoS. This is likely because RNAseq-based

214 approaches are can identify a broader range of gene transcripts as they are not limited by

215 restrictive microarray probes (42). Separate from differences in underlying technology, co-

216 expression networks are also more likely to detect genes regulating a target's expression

217 than traditional transcriptomics experiments which largely capture downstream responses

218 to changes in a target's expression $(43,44)$. Thus, a co-expression network can provide an

219 alternative perspective to complement or clarify transcriptomics data.

\section{Discussion}

222 We have successfully constructed the first $V$. cholerae co-expression network through a

223 computationally inexpensive process that is simple, easily expanded upon, and

224 straightforward to implement in other organisms. Our network effectively identifies

225 canonical gene clusters related to specific molecular pathways or functions, such as those

226 corresponding to rRNAs or biofilm proteins. We have also outlined two use-cases for the

227 data provided and have shown the accuracy of both approaches either experimentally or

228 using existing data. Additionally, we have included relevant network files as well as raw

229 read counts across RNA-seq conditions (Supp. Data S1-2 \& Supp. Table S2) alongside all 
230 code used in our analysis (see Materials and Methods) to encourage broad usage of this

231 data.

232 Our results have proven both the utility and accuracy of our approach despite in-depth

233 analysis limited to a handful of genes across five of the forty-nine observed gene clusters.

234 Furthermore, our work with the virulence subnetwork supports previously published

235 research loosely implicating genes VCA0094-96 in virulence and virulence related

236 functions. All three transcripts have shown up in screens focusing on biofilm development

237 (45), and SOS response (13). From a mechanistic perspective, protein homology analysis

238 via NCBI's Conserved Domain Database (46) indicates that VCA0094 possesses a DNA-

239 binding transcriptional regulator domain while VCA0096 contains domains that implicate

240 it in protein activation via proteolysis. These data combined with our novel findings hint at

241 the potential biological importance of this genomic locus.

242 When viewed through the lens of a specific gene of interest, co-expression data is in

243 large part analogous to the differential expression data produced by RNA-seq experiments.

244 While RNA-seq offers finer assay control and can be tailored more exactly to suit a specific

245 research question, there are both technical and practical limitations that may make such an

246 approach impractical. Whether an experimenter is interested in examining the role of an

247 essential locus or is limited by available resources, our co-expression analysis presents a

248 fast, free, and faithful alternative for probing genetic interactions as outlined in our analysis

249 of $r p o S$ above.

250 Major motivations for this work include the successful implementation of bacterial-

251 focus, microarray-based co-expression networks and the lack of clear functional knowledge

252 for a large portion of $V$. cholerae genes. Besides more simple guilt-by-association studies 
$253(22,23)$, co-expression networks have helped to elucidate relationships in diverse

254 microbial communities (47-50) and enable comparisons across strains and species (51-

255 53). These works as well as the relative dearth of knowledge about the $V$. cholerae genome

256 (roughly two third of genes are annotated compared to around 86\% percent of all E. coli

257 genes (54)) and the growing abundance of $V$. cholerae focused NGS data served as the

258 impetus for this research.

259 The calculated co-expression network, though accurate, could be improved via the

260 inclusion of more experiments and more extensive SRA annotations. Our somewhat limited

261 pooled dataset consisting of three hundred samples is an order of magnitude off from the

262 few thousand samples necessary to derive the most faithful co-expression estimates (55).

263 Though sample size will improve as more $V$. cholerae RNA-seq experiments are published,

264 more samples may also increase the risk posed by batch effects which cause spurious

265 correlations among genes through technical variation $(56,57)$. The diverse structure of our

266 current data helps to minimize the impact of batch effects but this would be offset by the

267 future inclusion of larger datasets from single experiments. While automated sample

268 clustering methods (58-60) can effectively group overly correlated samples, there is no

269 way to know if the correlation is biological (i.e. meaningful) or technical (i.e. noise) in

270 origin. Likewise, manual curation of batch annotations is also difficult since few SRA

271 records are extensively annotated with detailed experimental conditions (e.g. bacterial

272 growth stage, exact medium used). Thus, careful consideration may be necessary when

273 expanding and generalizing this analysis to include future data.

274 The mapping of raw reads to a transcriptome derived from a single reference genome

275 presents a limitation to our current work. While this approach is reasonable given the 
276 similarity of the vast majority of included strains to our reference, a more elaborate

277 comparative transcriptomic strategy $(61,62)$ would be ideal if more diverse samples are

278 included in future analyses. This is especially true when considering the inclusion of

279 expression data from clinical samples which are likely to have much more genomic

280 variability than the closely related lab cultured strains used to construct our network. On

281 the other hand, because comparative transcriptomics requires defining homologous alleles

282 across all strains analyzed (63), such an approach would greatly increase the difficulty of

283 incorporating strains without an assembled genome.

284 In summary, our co-expression network can drive functional hypotheses for

285 unannotated genes in V. cholerae. As the Vibrio community steadily adds high quality data

286 from increasingly sophisticated sequencing experiments to public databases our imputed

287 network can only improve, providing ever deeper insights into the $V$. cholerae genome. At

288 the same time, highly annotated transcript-based co-expression networks can empower

289 research with related technologies (e.g. single cell transcriptomics and dual RNA-seq) and

290 research into a host of other clinically relevant bacteria, such as Pseudomonas aeruginosa

291 or Staphylococcus aureus which have over 2000 and 1400 RNA-seq experiments in SRA

292 respectively.

\section{Materials and Methods}

295 Data Collection and Processing

296 All RNA and ChIP sequencing data were downloaded from the Sequence Read Archive

297 (SRA)(64) and converted to compressed fastq files using the SRA toolkit

298 (https://www.ncbi.nlm.nih.gov/sra/docs/toolkitsoft/) (see Table S1 for details on 
299 included experiments). RNA-seq samples were selected by searching the SRA on Sept $10^{\text {th }}$,

3002019 for the Organism and Strategy terms "vibrio cholerae" and "rna seq" respectively,

301 resulting in 326 initial samples including the 34 novel samples from this publication

302 (PRJNA601792). Samples were mapped to a recently inferred $V$. cholerae transcriptome

303 derived from the N16961 reference genome $(1,13)$ using Kallisto version 0.45 .1 (65). This

304 reference was chosen because the majority (293) of samples were collected from strains

305 N16961 or the closely related C6706 and A1552. 26 low quality samples with $<50 \%$ of

306 reads mapping to the reference transcriptome were discarded before further analysis,

307 leaving 300 samples used for further analysis.

308 For ChIP-seq analysis, accession numbers were identified via the relevant publications

$309(12,19,35)$ and sequences were downloaded from SRA and converted to fastq files as

310 above. Raw reads were mapped to the same N16961 reference genome using Bowtie 2

311 version 2.3.5.1 (66). From this mapping, peaks were identified using MACS2 version 2.1.2

312 with an extsize of 225 (various sizes from 150 to 500 were tested with little observable

313 difference in peaks identified) (67) and differential binding and significance were

314 calculated using DiffBind version 2.12.0 (68).

315 Processed Tn-seq data were collected directly from published datasets. In vitro

316 essentiality and semi-essentiality labels were derived from Chao et al. 2013 Table S1 (29),

317 with the original labels of domain essential and sick genes replaced with essential and

318 semi-essential respectively. We used Table S2 from Fu, Waldor, and Mekalanos 2013 (14)

319 to label genes involved in host infection, with any gene exhibiting a $\log _{2}$ fold change less

320 than negative three deemed essential and any gene with a $\log _{2}$ fold change between

321 negative one and negative three deemed semi-essential. 


\section{Network Construction}

323 Figure 1 highlights the process used to generate our co-expression network. Kallisto

324 derived reads were first imported into R via tximport (69), then normalized using DESeq2

325 version $1.24 .0(70)$, resulting in values that are comparable across conditions and

326 experiments. Following normalization, a weighted gene co-expression network analysis

327 was performed using WGCNA (21). This process is highlighted with a subset of data in

328 Figure 1 and consists of the sequential calculation of a Pearson correlation matrix,

329 adjacency matrix with power $\beta=6$, and, ultimately, topological overlap matrix (TOM) (24)

330 from normalized gene expression counts across conditions. We further filtered this TOM to

331 exclude samples with weighted co-expression $<0.1$ for all analysis included in the Results

332 section.

333 Predicted pathway annotations and gene functional knowledge are derived from the

334 NCBI Biosystems database as well as DAVID, Panther, and KEGG databases (25-28). Genes

335 lacking functional knowledge which are identified as essential or semi-essential in either

336 Tn-seq dataset are labeled in network visualizations as "important."

\section{Data Availability}

338 SRA accession numbers and information on included samples can be found in

339 Supplementary Table S1. A full, unfiltered network graph is provided in Supplementary File

340 S1 with the corresponding node labels in Supplementary File S2. Raw, un-normalized read

341 counts are also provided in Supplementary Table S2 All data analysis and figure generation

342 were done using the R programming language, with code available at DOI:

$34310.5281 /$ zenodo.3572870. 


\section{References}

347 1. Heidelberg JF, Eisen JA, Nelson WC, Clayton RA, Gwinn ML, Dodson RJ, Haft DH, Hickey

358 3. Greig DR, Schaefer U, Octavia S, Hunter E, Chattaway MA, Dallman TJ, Jenkins C. 2018. cholerae. J Clin Microbiol 56:e00831-18.

361 4. Domman D, Chowdhury F, Khan AI, Dorman MJ, Mutreja A, Uddin MI, Paul A, Begum YA, resolution within Bangladesh. Nat Genet 50:951-955.

365 5. Weill F-X, Domman D, Njamkepo E, Tarr C, Rauzier J, Fawal N, Keddy KH, Salje H, Moore 366 S, Mukhopadhyay AK, Bercion R, Luquero FJ, Ngandjio A, Dosso M, Monakhova E, Garin 
Mengel M, Koeck J-L, Fournier J-M, Dougan G, Grimont PAD, Parkhill J, Holt KE, Piarroux pandemic of cholera in Africa. Science (80- ) 358:785-789.

372 6. Domman D, Quilici M-L, Dorman MJ, Njamkepo E, Mutreja A, Mather AE, Delgado G, 373 Morales-Espinosa R, Grimont PAD, Lizárraga-Partida ML, Bouchier C, Aanensen DM, 374 Kuri-Morales P, Tarr CL, Dougan G, Parkhill J, Campos J, Cravioto A, Weill F-X, Thomson 375 NR. 2017. Integrated view of Vibrio cholerae in the Americas. Science 358:789-793.

376 7. Li Z, Pang B, Wang D, Li J, Xu J, Fang Y, Lu X, Kan B. 2019. Expanding dynamics of the 377 virulence-related gene variations in the toxigenic Vibrio cholerae serogroup 01. BMC $378 \quad$ Genomics 20:360.

379 8. Rahman MH, Biswas K, Hossain MA, Sack RB, Mekalanos JJ, Faruque SM. 2008. population in a cholera endemic area: tracking the evolution of pathogenic strains. DNA Cell Biol 27:347-355.

383 9. Lessler J, Moore SM, Luquero FJ, McKay HS, Grais R, Henkens M, Mengel M, Dunoyer J, M’bangombe M, Lee EC, Djingarey MH, Sudre B, Bompangue D, Fraser RSM, Abubakar A, (London, England) 391:1908-1915.

388 10. 2017. WHO | Ending Cholera. WHO.

389 11. Herzog R, Peschek N, Fröhlich KS, Schumacher K, Papenfort K. 2019. Three autoinducer molecules act in concert to control virulence gene expression in Vibrio cholerae. Nucleic Acids Res 47:3171-3183. 
392 12. Davies BW, Bogard RW, Young TS, Mekalanos JJ. 2012. Coordinated Regulation of

Accessory Genetic Elements Produces Cyclic Di-Nucleotides for V. cholerae Virulence. Cell 149:358-370.

13. Krin E, Pierlé SA, Sismeiro O, Jagla B, Dillies M-A, Varet H, Irazoki O, Campoy S, Rouy Z,

BMC Genomics 19:373.

14. Fu Y, Waldor MK, Mekalanos JJ. 2013. Tn-Seq Analysis of Vibrio cholerae Intestinal Host Microbe 14:652-663.

402 15. Mandlik A, Livny J, Robins WP, Ritchie JM, Mekalanos JJ, Waldor MK. 2011. RNA-Seqbased monitoring of infection-linked changes in Vibrio cholerae gene expression. Cell Host Microbe 10:165-174.

405 16. Kamp HD, Patimalla-Dipali B, Lazinski DW, Wallace-Gadsden F, Camilli A. 2013. Gene $406 \quad$ Fitness Landscapes of Vibrio cholerae at Important Stages of Its Life Cycle. PLoS Pathog 9:e1003800.

408 17. Pukatzki S, Ma AT, Sturtevant D, Krastins B, Sarracino D, Nelson WC, Heidelberg JF,

412 18. Kimura S, Hubbard TP, Davis BM, Waldor MK. 2016. The Nucleoid Binding Protein H-NS Biases Genome-Wide Transposon Insertion Landscapes. MBio 7:e01351-16.

414 19. Manneh-Roussel J, Haycocks JRJ, Magán A, Perez-Soto N, Voelz K, Camilli A, Krachler A- 
M, Grainger DC. 2018. cAMP Receptor Protein Controls Vibrio cholerae Gene Expression in Response to Host Colonization. MBio 9:e00966-18.

417 20. Saelens W, Cannoodt R, Saeys Y. 2018. A comprehensive evaluation of module detection 418 methods for gene expression data. Nat Commun 9:1090.

419 21. Langfelder P, Horvath S. 2008. WGCNA: an R package for weighted correlation network 420 analysis. BMC Bioinformatics 9:559.

421 22. Jiang J, Sun X, Wu W, Li L, Wu H, Zhang L, Yu G, Li Y. 2016. Construction and application 422 of a co-expression network in Mycobacterium tuberculosis. Sci Rep 6:28422.

423 23. Liu W, Li L, Long X, You W, Zhong Y, Wang M, Tao H, Lin S, He H. 2018. Construction and Analysis of Gene Co-Expression Networks in Escherichia coli. Cells 7:19.

425 24. Li A, Horvath S. 2006. Network neighborhood analysis with the multi-node topological overlap measure. Bioinformatics 23:222-231.

427 25. Geer LY, Marchler-Bauer A, Geer RC, Han L, He J, He S, Liu C, Shi W, Bryant SH. 2009. The NCBI BioSystems database. Nucleic Acids Res 38:D492-D496.

429 26. Huang DW, Sherman BT, Lempicki RA. 2008. Systematic and integrative analysis of large gene lists using DAVID bioinformatics resources. Nat Protoc 4:44.

431 27. Kanehisa M, Goto S. 2000. KEGG: kyoto encyclopedia of genes and genomes. Nucleic Acids Res 28:27-30.

433 28. Mi H, Dong Q, Muruganujan A, Gaudet P, Lewis S, Thomas PD. 2009. PANTHER version 7: 434 improved phylogenetic trees, orthologs and collaboration with the Gene Ontology $435 \quad$ Consortium. Nucleic Acids Res 38:D204-D210.

436 29. Chao MC, Pritchard JR, Zhang YJ, Rubin EJ, Livny J, Davis BM, Waldor MK. 2013. Highresolution definition of the Vibrio cholerae essential gene set with hidden Markov 
model-based analyses of transposon-insertion sequencing data. Nucleic Acids Res 41:9033-9048.

440 30. van Dam S, Võsa U, van der Graaf A, Franke L, de Magalhães JP. 2017. Gene co-expression analysis for functional classification and gene-disease predictions. Brief Bioinform 19:575-592.

443 31. Silva AJ, Benitez JA. 2016. Vibrio cholerae Biofilms and Cholera Pathogenesis. PLoS Negl Trop Dis 10:e0004330.

445 32. Teschler JK, Zamorano-Sánchez D, Utada AS, Warner CJA, Wong GCL, Linington RG, Yildiz FH. 2015. Living in the matrix: assembly and control of Vibrio cholerae biofilms. Nat Rev

448 33. Papenfort K, Förstner KU, Cong J-P, Sharma CM, Bassler BL. 2015. Differential RNA-seq of Vibrio cholerae identifies the VqmR small RNA as a regulator of biofilm formation. Proc Natl Acad Sci 112:E766-E775.

451 34. Weber GG, Klose KE, Klose. 2011. The complexity of ToxT-dependent transcription in Vibrio cholerae. Indian J Med Res 133:201-6.

453 35. Kazi MI, Conrado AR, Mey AR, Payne SM, Davies BW. 2016. ToxR Antagonizes H-NS 454 Regulation of Horizontally Acquired Genes to Drive Host Colonization. PLOS Pathog 12:e1005570.

456 36. Dorman MJ, Dorman CJ. 2018. Regulatory Hierarchies Controlling Virulence Gene Expression in Shigella flexneri and Vibrio cholerae. Front Microbiol.

458 37. Ayala JC, Wang H, Silva AJ, Benitez JA. 2015. Repression by H-NS of genes required for 459 the biosynthesis of the Vibrio cholerae biofilm matrix is modulated by the second 460 messenger cyclic diguanylic acid. Mol Microbiol 97:630-645. 
461 38. Boyd EF, Jermyn WS. 2002. Characterization of a novel Vibrio pathogenicity island (VPIMicrobiology 148:3681-3693.

464 39. Karaolis DKR, Johnson JA, Bailey CC, Boedeker EC, Kaper JB, Reeves PR. 1998. A Vibrio cholerae pathogenicity island associated with epidemic and pandemic strains. Proc Natl

467 40. Nielsen AT, Dolganov NA, Otto G, Miller MC, Wu CY, Schoolnik GK. 2006. RpoS controls the Vibrio cholerae mucosal escape response. PLoS Pathog 2:e109-e109.

469 41. Wong GT, Bonocora RP, Schep AN, Beeler SM, Lee Fong AJ, Shull LM, Batachari LE, Dillon

473 42. Russo G, Zegar C, Giordano A. 2003. Advantages and limitations of microarray technology in human cancer. Oncogene 22:6497-6507.

475 43. Serin EAR, Nijveen H, Hilhorst HWM, Ligterink W. 2016. Learning from Co-expression Networks: Possibilities and Challenges. Front Plant Sci 7:444.

477 44. Koschmann J, Bhar A, Stegmaier P, Kel AE, Wingender E. 2015. "Upstream Analysis”: An Data. Microarrays (Basel, Switzerland) 4:270-286.

480 45. Mueller RS, Mcdougald D, Cusumano D, Sodhi N, Kjelleberg S, Azam F, Bartlett DH. 2007. Colonization. J Bacteriol 189:5348-5360.

483 46. Marchler-Bauer A, Bo Y, Han L, He J, Lanczycki CJ, Lu S, Chitsaz F, Derbyshire MK, Geer 
RC, Gonzales NR, Gwadz M, Hurwitz DI, Lu F, Marchler GH, Song JS, Thanki N, Wang Z, classification of proteins via subfamily domain architectures. Nucleic Acids Res 45:D200-D203.

488 47. Duran-Pinedo AE, Paster B, Teles R, Frias-Lopez J. 2011. Correlation Network Analysis Applied to Complex Biofilm Communities. PLoS One 6:e28438.

48. Geng H, Tran-Gyamfi MB, Lane TW, Sale KL, Yu ET. 2016. Changes in the Structure of the Microbial Community Associated with Nannochloropsis salina following Treatments with Antibiotics and Bioactive Compounds. Front Microbiol 7:1155.

493 49. Meisel JS, Sfyroera G, Bartow-McKenney C, Gimblet C, Bugayev J, Horwinski J, Kim B,

496 50. Jackson MA, Bonder MJ, Kuncheva Z, Zierer J, Fu J, Kurilshikov A, Wijmenga C, structures within gut microbiota co-occurrence networks from different human populations. PeerJ 6:e4303.

500 51. Hosseinkhan N, Mousavian Z, Masoudi-Nejad A. 2018. Comparison of gene co-expression networks in Pseudomonas aeruginosa and Staphylococcus aureus reveals conservation in some aspects of virulence. Gene 639:1-10.

503 52. Peña-Castillo L, Mercer RG, Gurinovich A, Callister SJ, Wright AT, Westbye AB, Beatty JT, $504 \quad$ Lang AS. 2014. Gene co-expression network analysis in Rhodobacter capsulatus and 505 application to comparative expression analysis of Rhodobacter sphaeroides. BMC $506 \quad$ Genomics 15:730. 
507 53. Wang J, Wu G, Chen L, Zhang W. 2013. Cross-species transcriptional network analysis reveals conservation and variation in response to metal stress in cyanobacteria. BMC Genomics 14:112.

510 54. Keseler IM, Mackie A, Santos-Zavaleta A, Billington R, Bonavides-Martínez C, Caspi R, Fulcher C, Gama-Castro S, Kothari A, Krummenacker M, Latendresse M, Muñiz-Rascado

515 55. Ballouz S, Verleyen W, Gillis J. 2015. Guidance for RNA-seq co-expression network Vides J, Paulsen I, Karp PD. 2017. The EcoCyc database: reflecting new knowledge about

517 56. Li S, Tighe SW, Nicolet CM, Grove D, Levy S, Farmerie W, Viale A, Wright C, Schweitzer PA, Gao Y, Kim D, Boland J, Hicks B, Kim R, Chhangawala S, Jafari N, Raghavachari N, Escherichia coli K-12. Nucleic Acids Res2016/11/29. 45:D543-D550.

523 57. Goh WW Bin, Wang W, Wong L. 2017. Why Batch Effects Matter in Omics Data, and How assessment of transcriptome profiling using RNA-seq in the ABRF next-generation sequencing study. Nat Biotechnol 32:915-925.

525 58. Leek JT, Storey JD. 2007. Capturing heterogeneity in gene expression studies by surrogate variable analysis. PLoS Genet 3:1724-1735.

527 59. Alter 0, Brown PO, Botstein D. 2000. Singular value decomposition for genome-Wide expression data processing and modeling. Proc Natl Acad Sci U S A 97:10101-10106.

529 60. Leek JT, Scharpf RB, Bravo HC, Simcha D, Langmead B, Johnson WE, Geman D, Baggerly 
K, Irizarry RA. 2010. Tackling the widespread and critical impact of batch effects in highthroughput data. Nat Rev Genet.

532 61. Cohen O, Doron S, Wurtzel O, Dar D, Edelheit S, Karunker I, Mick E, Sorek R. 2016. Comparative transcriptomics across the prokaryotic tree of life. Nucleic Acids Res 44:W46-W53.

62. Chang YM, Lin HH, Liu WY, Yu CP, Chen HJ, Wartini PP, Kao YY, Wu YH, Lin JJ, Lu MYJ, Tu SL, Wu SH, Shiu SH, Ku MSB, Li WH. 2019. Comparative transcriptomics method to infer gene coexpression networks and its applications to maize and rice leaf transcriptomes. Proc Natl Acad Sci U S A 116:3091-3099.

63. Rodríguez-García A, Sola-Landa A, Barreiro C. 2017. RNA-Seq-Based Comparative Transcriptomics: RNA Preparation and Bioinformatics BT - Microbial Steroids: Methods and Protocols, p. 59-72. In Barredo, J-L, Herráiz, I (eds.), . Springer New York, New York,

543 64. Leinonen R, Sugawara H, Shumway M, Collaboration INSD. 2011. The sequence read archive. Nucleic Acids Res2010/11/09. 39:D19-D21.

545 65. Bray NL, Pimentel H, Melsted P, Pachter L. 2016. Near-optimal probabilistic RNA-seq 546 quantification. Nat Biotechnol 34:525-527.

547 66. Langmean B, Salzberg SL. 2012. Bowtie 2. Nat Methods 9:357-359.

548 67. Gaspar JM. 2018. Improved peak-calling with MACS2. bioRxiv 496521.

549 68. Ross-Innes CS, Stark R, Teschendorff AE, Holmes KA, Ali HR, Dunning MJ, Brown GD, 550 Gojis O, Ellis IO, Green AR, Ali S, Chin S-F, Palmieri C, Caldas C, Carroll JS. 2012.

551 Differential oestrogen receptor binding is associated with clinical outcome in breast $552 \quad$ cancer. Nature 481:389. 
553 69. Soneson C, Love MI, Robinson MD. 2015. Differential analyses for RNA-seq: transcriptlevel estimates improve gene-level inferences. F1000Research 4:1521.

555 70. Love MI, Huber W, Anders S. 2014. Moderated estimation of fold change and dispersion for RNA-seq data with DESeq2. Genome Biol 15:550.

558 Legends:

559 Figure 1: General outline of network construction.

$5601 \mathrm{~A})$ Normalized $(\log 2)$ expression reads for the same genes across multiple conditions supply

561 the basis for our co-expression analysis. In this small example, it is clear that genes VC0384-

562 VC0386 have a very similar expression pattern across conditions. 1B) Correlations are

563 calculated from the normalized counts in A for every pair of genes. The pattern seen in A

564 becomes much clearer when looking at the correlation. 1C) An adjacency matrix (not shown) is

565 calculated from the correlations in B and ultimately used to produce a topological overlap

566 matrix (TOM) that supplies network edge weights with less noise than the raw correlation

567 matrix. While the single of co-expressing pairs is dampened slightly, this step greatly decreases

568 spurious relationships as it favors transcripts which coexpress with similar sets of genes

569 rather than potentially noisy direct correlations. 1D) The final network groups transcripts that

570 tightly co-express while indicating what pathway they are involved in. This network also

571 includes functional and essentiality based knowledge. In this case, the three genes involved in

572 cysteine metabolism (VC0383-VC0385, cysHI) form a subnetwork while the other genes do

573 not meet our 0.10 co-expression cutoff.

575 Figure 2: Sub-networks recapitulating known results 
576 The four depicted subnetworks each contain subsets of transcripts which are known to be

577 largely involved in the same biological process. For each subnetwork, the nodes represent

578 transcripts while the edges represent a co-expression relationship of at least 0.1 between

579 transcripts. A) This sub-network consists completely of tRNA transcripts. B) These transcripts

580 are almost completely related to ribosomal structure and/or function. C) These transcripts

581 play a role in amino acid synthesis. D) This sub-network contains a majority of transcripts that

582 play a role in biofilm formation in addition to unrelated genes.

583

584 Figure 3: Virulence related subnetwork.

5853 3) This subnetwork contains a majority of genes that are predicted to be involved in

586 virulence related pathways, providing clues to the genes with no known functions such as

587 those at locus VCA0094-VCA0096. 3B) Mean binding affinity (log2 fold change in occupancy

588 compared to loading control) for different virulence-associated transcription factors near the

589 VCA0094-96 locus. Both HNS and TOXR show a significant binding preference for this region.

590 Error bars indicate standard deviation from the mean.

592 Figure 4: Comparing RpoS microarray data to co-expressing genes in our WGCNA

593 A) Overlap of genes with expression pattern related to rpoS expression as identified via our

594 network analysis (blue) and existing microarray data (red). The overlapping region identifies

595272 genes that are common between the two analyses. B) Breakdown of shared genes

596 (overlapping region in A). All of the flagellar and chemotaxis genes highlighted as particularly

597 important in the microarray dataset are identified by both methods. 
bioRxiv preprint doi: https://doi.org/10.1101/2020.02.07.939611; this version posted February 9,2020 . The copyright holder for this preprint (which was not certified by peer review) is the author/funder, who has granted bioRxiv a license to display the preprint in perpetuity. It is made available under aCC-BY 4.0 International license.

600

\section{Figure 1}

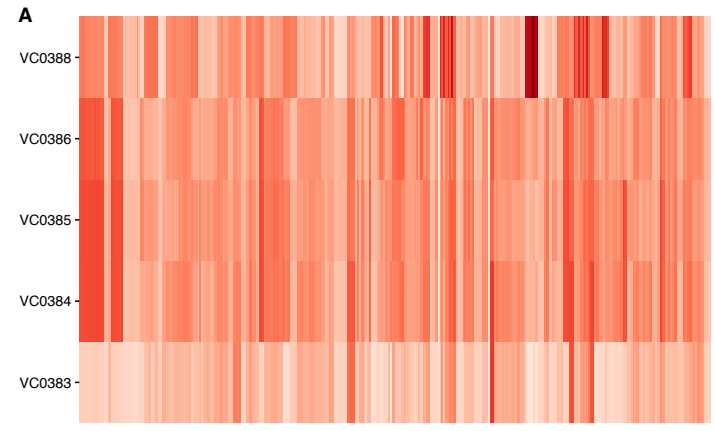

Condition

C

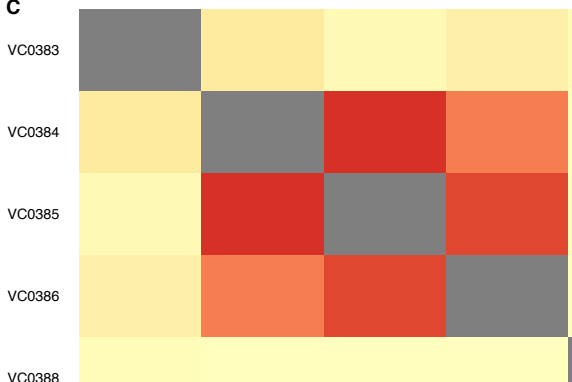

602

603

604

605

606

607

608

609

610

611

612

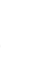

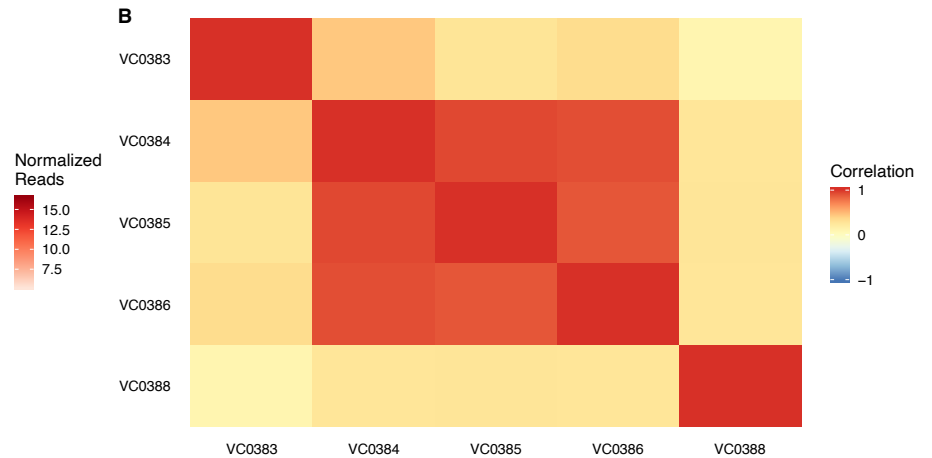

D

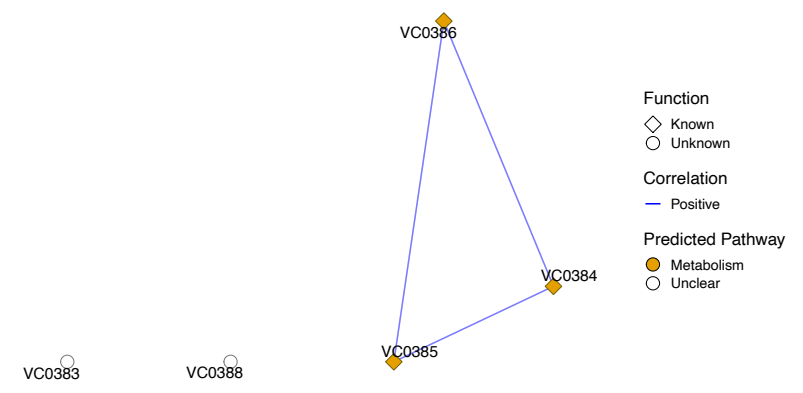

vc0388
Predicted Pathway

O Metabolism

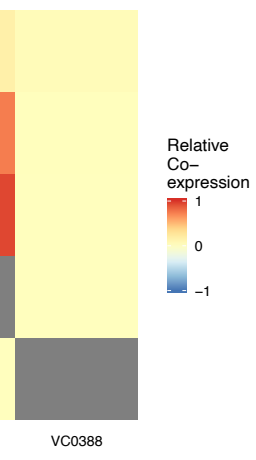

vс0383 
bioRxiv preprint doi: https://doi. org/10.1101/2020.02.07 939611. this version posted February 92020 . The copyright holder for this preprint (which was not certified by peer review) is the author/funder, who has granted bioRxiv a license to display the preprint in perpetuity. It is made available under aCC-BY 4.0 International license.

615 Figure 2

\section{A tRNA Transcripts}

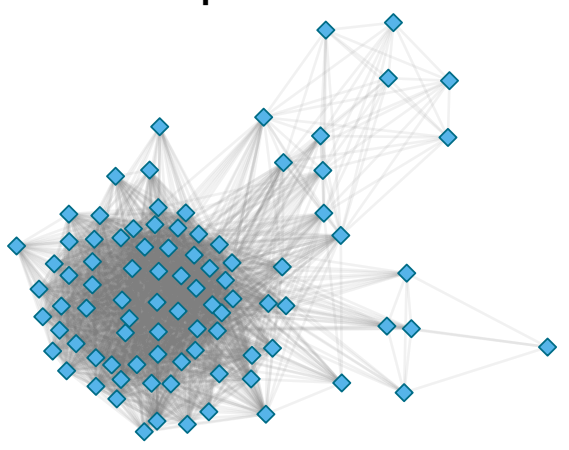

C Amino Acid Synthesis

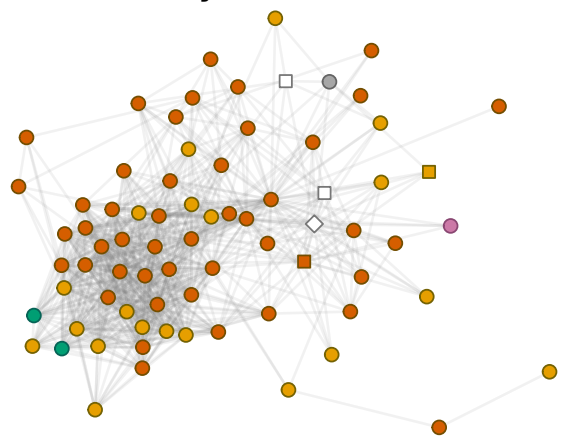

B Ribosome Related

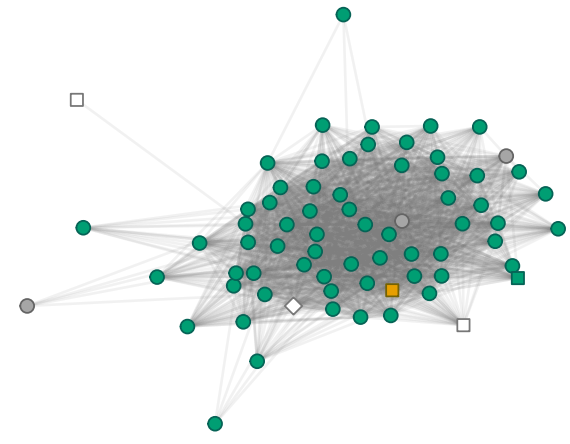

D Biofilm

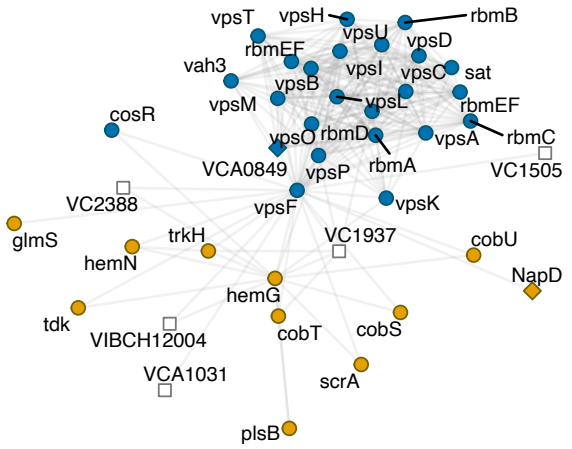

Predicted Pathway

- Amino Acid Synthes

- Biofilm

○ Metabolism

- Ribosome

- RNA/DNA

O tRNA

O Unclear

- Virulence

Function

$\diamond \quad$ Important

O Known

Unknown 
bioRxiv preprint doi: $h t t p s: / / d o i . o r g / 10.1101 / 2020.0207939611^{\cdot}$ this version posted February 92020 . The copyright holder for this preprint (which was not certified by peer review) is the author/funder, who has granted bioRxiv a license to display the preprint in perpetuity. It is made available under aCC-BY 4.0 International license.

\section{Figure 3}

A

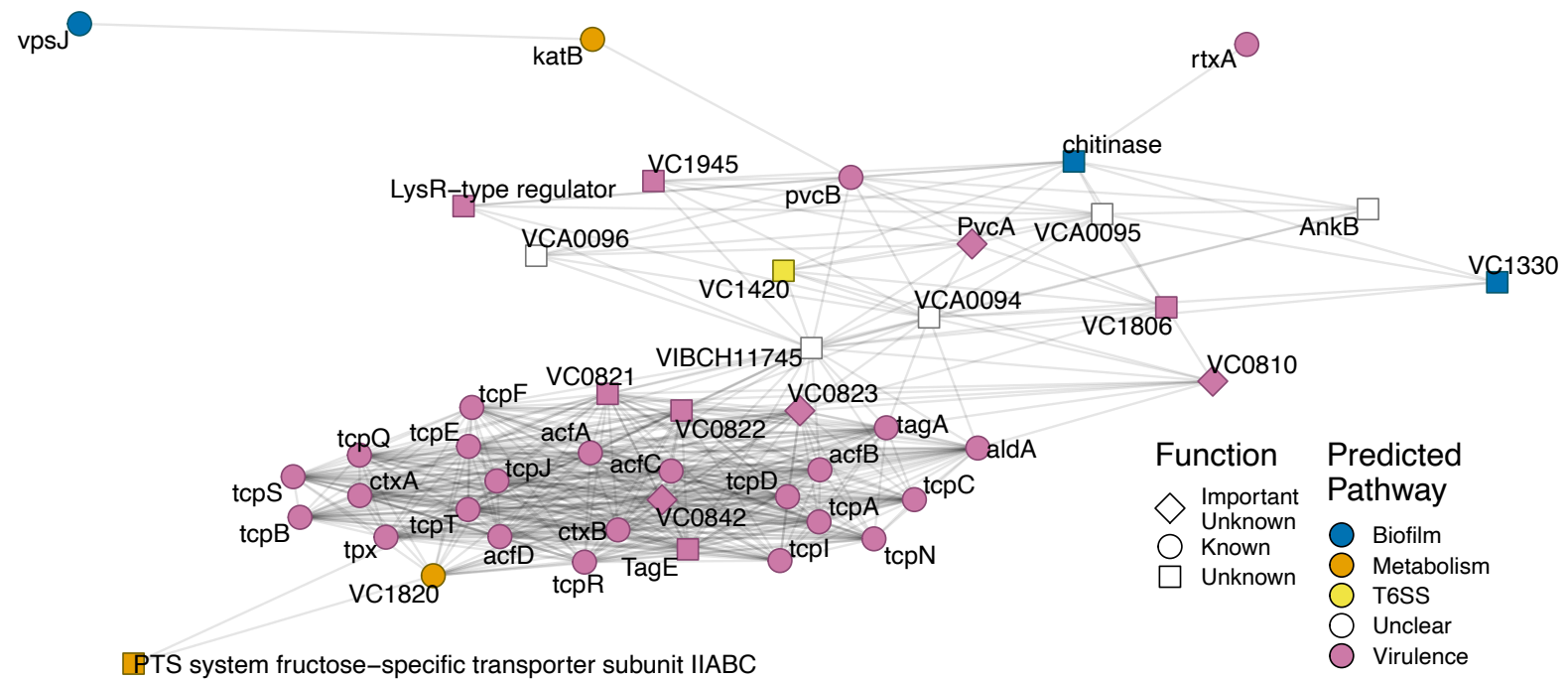

B

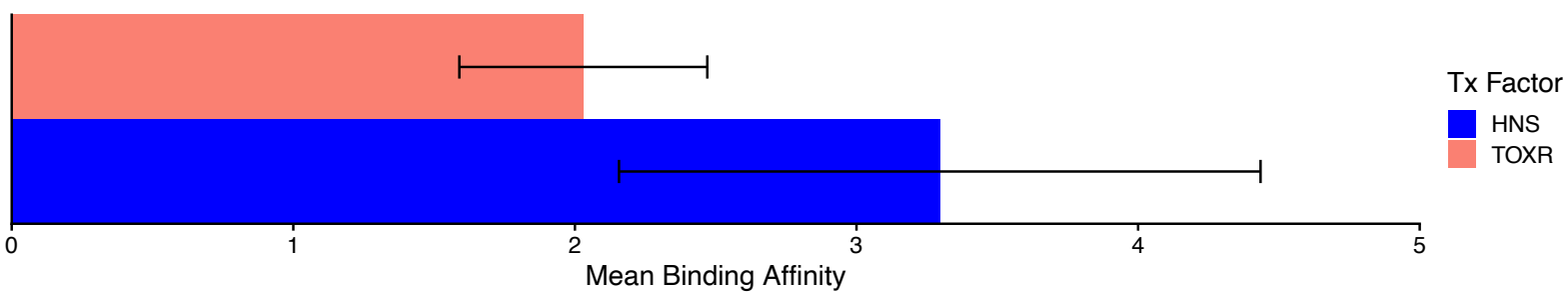


bioRxiv preprint doi: https://doi.org/10.1101/2020.02.07.939611; this version posted February 9, 2020. The copyright holder for this preprint (which was not certified by peer review) is the author/funder, who has granted bioRxiv a license to display the preprint in perpetuity. It is made available under aCC-BY 4.0 International license.

636

637

\section{Figure 4}

A

B

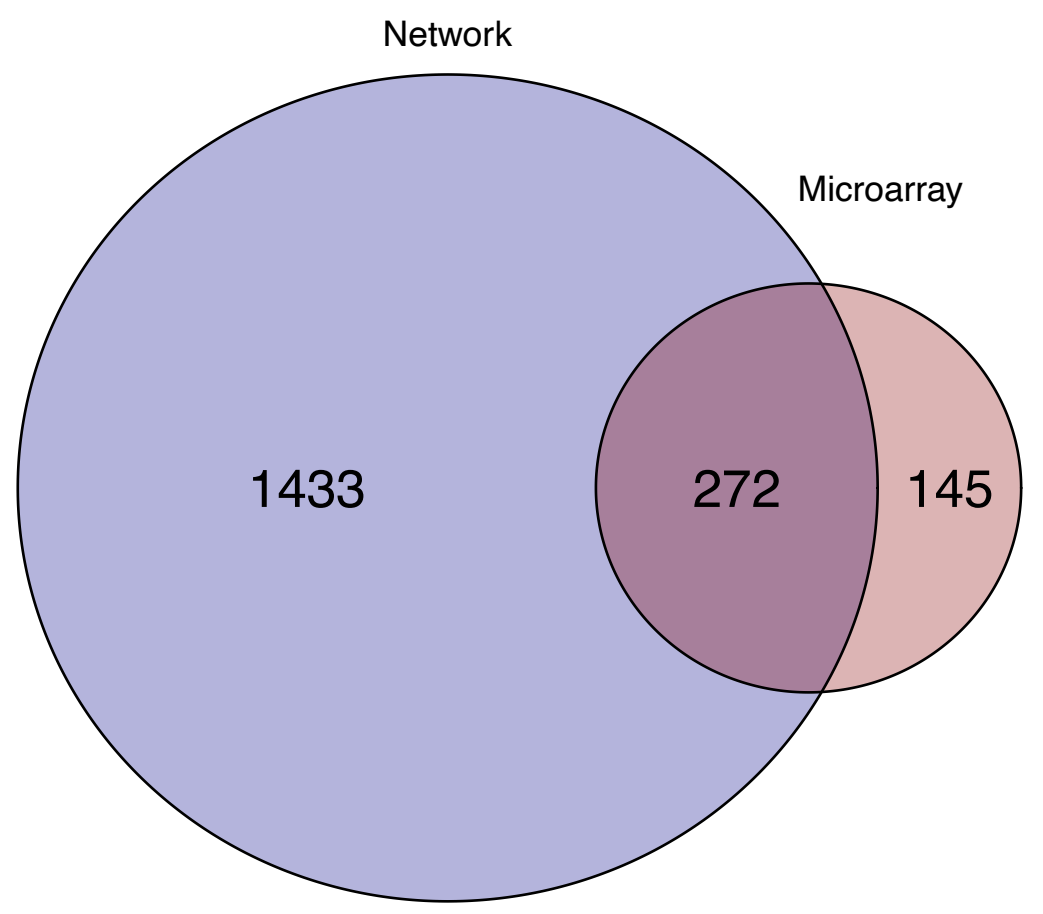

$\square$ Other $\square$ Flagellar/Chemotaxis

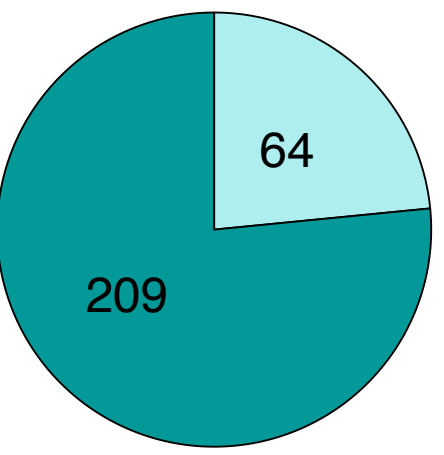

